\title{
Abundance analysis of seven $\delta$ Scuti stars
}

\author{
L. Fossati ${ }^{1}$, K. Kolenberg ${ }^{1,2}$, P. Reegen ${ }^{1}$, and W. Weiss ${ }^{1}$ \\ 1 Institut fur Astronomie, Universität Wien, Türkenschanzstraße 17, 1180 Wien, Austria \\ e-mail: [fossati; reegen; weiss] aastro.univie.ac.at \\ 2 Instituut voor Sterrenkunde, Celestijnenlaan 200D, 3001 Leuven, Belgium \\ e-mail: kolenberg@astro.univie.ac.at
}

Received 8 February 2008 / Accepted 21 April 2008

\begin{abstract}
Context. The current knowledge of the abundance pattern in $\delta$ Scuti stars is based on analysis of just a few field stars.

Aims. We aim to determine the general chemical properties of the atmospheres of $\delta$ Scuti stars based on a statistically relevant sample of stars and investigate whether the abundance pattern is close to solar, an assumption generally made for pulsation models.

Methods. We analysed high-resolution, high signal-to-noise ratio spectra of seven field $\delta$ Scuti stars. We derived the fundamental parameters and the photospheric abundances and than compared them to a similar sample of cluster $\delta$ Scuti stars.

Results. With the use of a $t$-test we demonstrate that there is no difference between the two samples, which allows us to merge them, resulting in a sample of fifteen $\delta$ Scuti stars. We do not find any substantial difference between the abundance pattern of our sample of $\delta$ Scuti stars and a sample of normal early A- and late F-type stars. One field star in our sample, HD 124953, is most likely a pulsating Am star.
\end{abstract}

Key words. stars: abundances - stars: atmospheres - delta Sct

\section{Introduction}

It is widely accepted that chemical peculiarity and pulsation are almost mutually exclusive. The diffusion hypothesis (Vauclair et al. 1978) explains in detail why this occurs. The $\delta$ Scuti pulsation is driven by the $\kappa$ mechanism in the He II ionisation zone. For stars in which helium is gravitationally settled due to diffusion, the driving mechanism is less efficient and pulsation is inhibited. Nevertheless, there are a few chemically peculiar Am and Ap stars that do pulsate. It is thought that, in these stars, the pulsation is laminar enough not to disrupt the anomalous distribution of elements.

Since the He II ionisation zone drives the pulsation, it is expected that the photospheric abundances of the individual elements have a negligible influence on the pulsation properties. In other words, the specific "skin diseases" (Breger et al. 2006) of some $\delta$ Scuti stars may not be relevant for modelling their pulsations. However, it is only through detailed analyses that we can quantify the element abundances (diagnose the skin disease, to stay with the analogue).

Throughout the literature, only a few detailed abundance analyses of $\delta$ Scuti stars can be found (e.g. Mittermayer \& Weiss 2002; Zima et al. 2007; Bruntt et al. 2008). In this paper, we present one of the few detailed element abundance analyses available for $\delta$ Scuti stars and the first of a statistically relevant sample of stars. We carried out spectroscopic abundance analyses for seven field $\delta$ Scuti stars. We want to answer the question whether the abundance pattern of field $\delta$ Scuti stars is substantially different from those of cluster $\delta$ Scuti stars, such as the stars in the Praesepe cluster analysed by Fossati et al. (2007) and Fossati et al. (2008), and how the abundance pattern of $\delta$ Scuti stars compares with those of normal F- and A-type stars.
The current analysis is part of a large project carried out by the authors and several other collaborators that aims at the analysis of chemical (see e.g. Fossati et al. 2008) and magnetic (see Bagnulo et al. 2006; Landstreet et al. 2007, 2008) properties of early-type stars in open clusters.

Breger (2000) pointed out that "about $50 \%$ of all main sequence stars inside the instability strip are $\delta$ Scuti pulsators" and that probably the other $50 \%$ "do pulsate with amplitudes below the present level of detection". Within this context our analysis turns out to also be an abundance analysis of non-chemically peculiar (CP: Fm, Am, $\lambda$ Boo and Ap stars) early F- and late A-type field stars.

The observed stars, adopted instrumentation and the abundance analysis procedure are described in Sect. 2. In Sect. 3 we present our results including a description of the pulsation characteristics for each star of our sample. Discussion and conclusion are given in Sects. 4 and 5, respectively.

\section{Observations, data reduction and abundance analysis}

We observed seven $\delta$ Scuti stars, present in the catalogue of Rodríguez et al. (2000) with the SOPHIE spectrograph at the Observatoire de Haute-Provence (OHP) from 2007 March 10-12. SOPHIE is a cross-dispersed échelle spectrograph mounted on the $1.93-\mathrm{m}$ telescope at the OHP. The spectrograph is fed from the Cassegrain focus through pairs of optical fibers, one of which is used for starlight and the other can be used for either the wavelength calibration lamp or the sky background, but can also be masked. The spectra cover the wavelength range 3872-6943 $\AA$ and the instrument allows 
Table 1. Basic data for the observations and the pulsation properties of the program stars.

\begin{tabular}{rrrrrrrrr}
\hline \hline HD & HJD & $\begin{array}{r}M_{v} \\
{[\mathrm{mag}]}\end{array}$ & Spectral type & $S N R$ & $\begin{array}{r}\text { Exp. time } \\
{[\mathrm{s}]}\end{array}$ & $\log P$ & $\begin{array}{r}Q \\
{[\mathrm{~d}]}\end{array}$ & Remarks \\
\hline 127929 & 2454170.650 & 6.27 & FOIII & 238 & 550 & -1.06 & 0.018 & \\
138918 & 2454170.693 & 3.80 & FOIV & 286 & 550 & -0.81 & 0.032 & binary \\
143466 & 2454171.703 & 4.97 & F0IV & 465 & 550 & -1.12 & 0.029 & \\
124675 & 2454170.598 & 4.74 & A8IV & 390 & 600 & -1.19 & 0.015 & binary \\
124953 & 2454170.614 & 5.97 & A8III & 257 & 550 & & & binary? \\
125161 & 2454170.626 & 4.75 & A9V & 295 & 550 & -1.58 & 0.013 & \\
127762 & 2454170.686 & 3.00 & A7III & 334 & 550 & -1.14 & 0.017 & binary \\
\hline
\end{tabular}

The $S N R$ is calculated at $\sim 5500 \AA$ in a bin of $0.5 \AA$. The exposure time is in seconds. The HJD indicates the Heliocentric Julian Date at the end of the exposure. The analysed spectrum of HD 127929 is the sum of two spectra with the same exposure time. Periods and $Q$ values are taken from Rodríguez et al. (2000) and López de Coca et al. (1990).

either mid-resolution mode $(R \sim 40000)$ or high-resolution mode $(R \sim 75000)$. All the stars of our sample were observed in high resolution. The spectra were automatically reduced using a pipeline adapted from the HARPS software designed by Geneva Observatory (see the SOPHIE web page ${ }^{1}$ ).

The sample of stars observed and analysed in this paper are listed in Table 1. Three stars are F-type, while the other four are A-type stars. Almost all the stars in the sample have a high projected rotational velocity $\left(v \sin i \geq 50 \mathrm{~km} \mathrm{~s}^{-1}\right)$. For these objects the continuum normalisation is crucial and it was performed without the use of any automatic continuum fitting process. First we divided the spectra in portions of about $700 \AA$ each. Subsequently, we performed the normalisation by fitting a spline to carefully selected continuum windows. These were identified by comparison with a synthetic spectrum of the same approximate $T_{\text {eff }}, \log g$ and $v \sin i$. It was not possible to determine a correct continuum level at wavelengths shorter than the $\mathrm{H} \gamma$ line (4340 $\AA$ ) since there are not enough continuum windows in the spectra because of the crowding of spectral lines in this region.

Model atmospheres were calculated with LLMODELS, an LTE code which uses direct sampling of the line opacities (Shulyak et al. 2004) and allows computing models with an individualised abundance pattern. Spectral line data were extracted from the VALD database (Piskunov et al. 1995; Kupka et al. 1999; Ryabchikova et al. 1999).

The fundamental parameters were derived spectroscopically using as starting models those from Strömgren and Geneva photometry, adopting calibrations from Napiwotzki et al. (1993) and Kunzli et al. (1997). Effective temperature and $\log g$ were obtained from Fe I and Fe II lines and from fitting the $\mathrm{H} \alpha$ line wings. For most of the échelle spectrographs it is very difficult to use the Balmer lines to constrain the fundamental parameters, since the orders are not large enough to completely include the wings. With the SOPHIE spectrograph, however, it is possible due to the reduction pipeline which rescales and merges the orders before the normalisation. In this way, a single spectrum is obtained (shown in Fig. 1) that allows the normalisation of the Balmer lines. We adopted $\mathrm{H} \alpha$ for the determination of $T_{\text {eff }}$ since it has several continuum points close to the wings. An example of the fit to an $\mathrm{H} \alpha$ line is given in Fig. 2. The plot demonstrates that we can determine $T_{\text {eff }}$ with an uncertainty of about $200 \mathrm{~K}$. This method is only weakly dependent on the projected rotational velocity $(v \sin i)$. In most cases the photometric and spectroscopic temperatures were consistent.

${ }^{1}$ www.obs-hp.fr/www/guide/sophie/sophie-eng.html

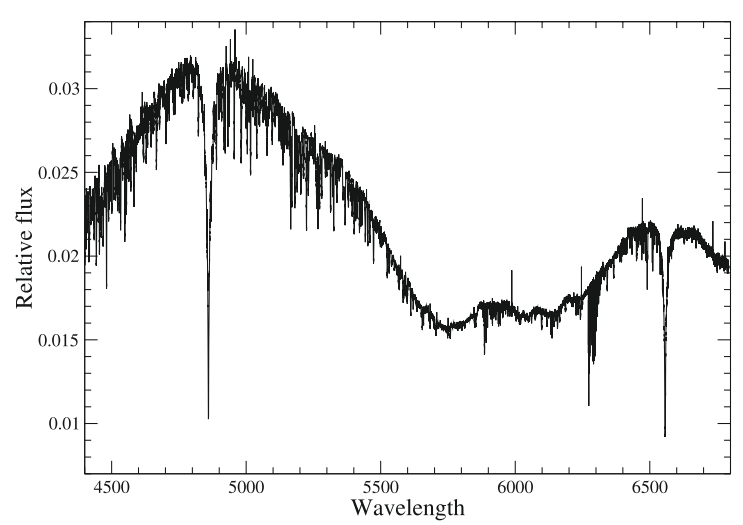

Fig. 1. The spectrum of HD 124953 from the SOPHIE reduction pipeline.

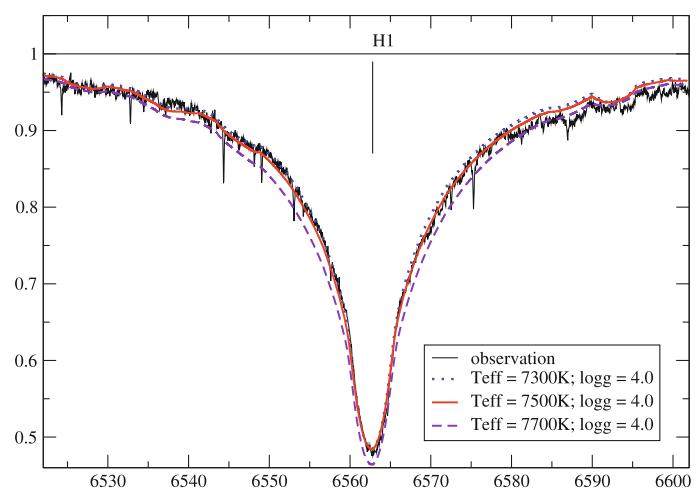

Fig. 2. $\mathrm{H} \alpha$ line fitting for $\mathrm{HD} 124675\left(v \sin i=121 \mathrm{~km} \mathrm{~s}^{-1}\right)$ to estimate the effective temperature. The three plotted synthetic spectra correspond to $T_{\text {eff }}$ of $7300 \mathrm{~K}$ (dotted line), $7500 \mathrm{~K}$ (full line) and $7700 \mathrm{~K}$ (dashed line).

Bruntt et al. (2008) showed that the uncertainty on the spectroscopically derived $\log g$ value is dependent on $v \sin i$. Their Table 3 shows the increment of the uncertainty on $\log g$ with increasing $v \sin i$ from $10 \mathrm{~km} \mathrm{~s}^{-1}$ to $60 \mathrm{~km} \mathrm{~s}^{-1}$. A linear extrapolation of this uncertainty at $v \sin i=110 \mathrm{~km} \mathrm{~s}^{-1}$ (about the mean $v \sin i$ in our sample) leads to an error bar on $\log g$ of about 0.22 dex. We derived $v_{\text {mic }}$ as in Fossati et al. (2008) and assumed the same uncertainty. Therefore the uncertainties on $T_{\text {eff }}, \log g$ and $v_{\text {mic }}$ are estimated to be $200 \mathrm{~K}, 0.22 \mathrm{dex}$ and $0.7 \mathrm{~km} \mathrm{~s}^{-1}$, respectively.

An example of the observed spectra of the moderate rotator HD $124953\left(v \sin i=82 \mathrm{~km} \mathrm{~s}^{-1}\right)$ and the fast rotator HD 124675 

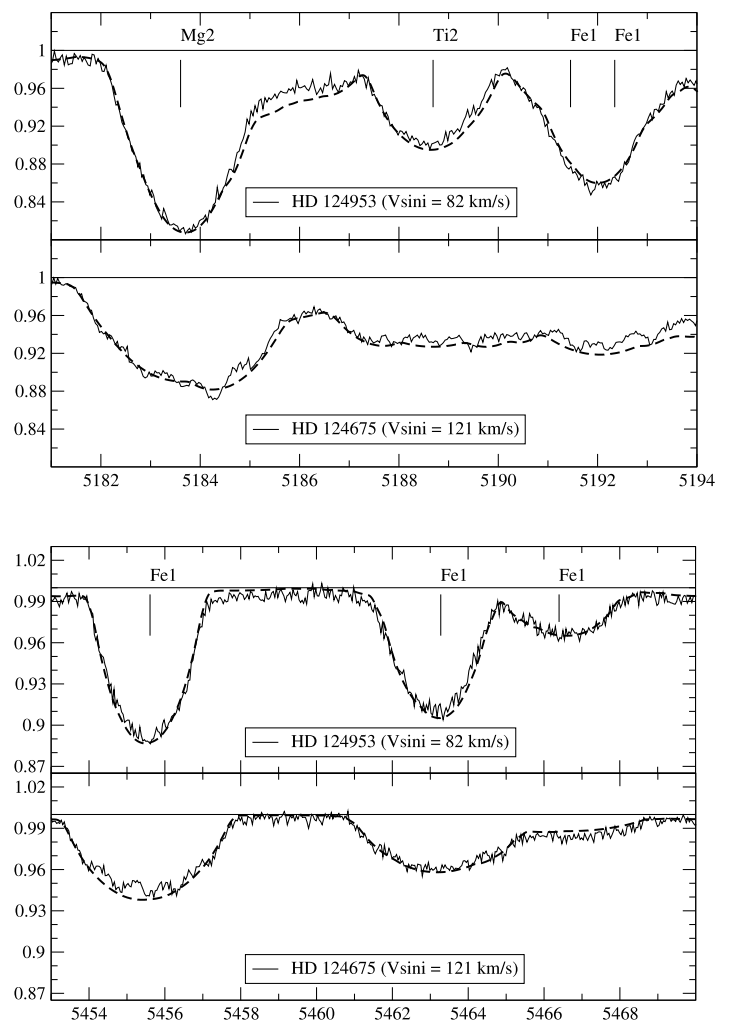

Fig. 3. Portions of the observed spectra of HD $124953(v \sin i=$ $82 \mathrm{~km} \mathrm{~s}^{-1}$, upper panels $)$ and of HD $124675\left(v \sin i=121 \mathrm{~km} \mathrm{~s}^{-1}\right.$, lower panels) and the synthetic spectra are shown with full and dashed lines, respectively.

( $v \sin i=121 \mathrm{~km} \mathrm{~s}^{-1}$ ) and the synthetic spectra obtained from the abundance analysis is shown in Fig. 3.

Details on the parameter determination and on the abundance analysis method including a thorough discussion of the abundance uncertainties for fast rotating stars are presented in Fossati et al. (2007, 2008).

We adopted the results by Fossati et al. (2008) for the estimation of the abundance uncertainties, which are affected by fast rotation of the stars and the uncertainties on $T_{\text {eff }}$ and $v_{\text {mic }}$. For the stars in our sample the uncertainty is about 0.25 dex.

Another source of uncertainty, which is dependent on $v \sin i$ is the continuum normalisation. To quantify this uncertainty we performed the following test. We derived the abundance of the Fe II line at 5325.6 $\AA$ for HD $124953(v \sin i=$ $82 \mathrm{~km} \mathrm{~s}^{-1}$ ) with the adopted normalised observed spectrum and with the spectrum multiplied/divided by 0.99 . In this way we increased/decreased the continuum level by $1 \%$, which we estimate to be a reasonable uncertainty. The difference between the abundances obtained in this way is about $0.1 \mathrm{dex}$, consistent with what was obtained with the same experiment by Fossati et al. (2008). Their conclusion is that the abundance error bar due to the uncertainty on the continuum normalisation is increasing with $v \sin i$ up to $0.2 \mathrm{dex}$ for stars with $v \sin i \sim 200 \mathrm{~km} \mathrm{~s}^{-1}$. We believe that in most cases this error bar is an upper limit since the line-by-line abundance analysis method, used in this work, allows a very careful line selection that rejects all the lines for which the continuum level looks uncertain. Under the assumption that no systematic errors are present the uncertainty will decrease when several lines are used for an element. For a more thorough evaluation see Erspamer \& North (2003) who showed how this source of error depends on the element.
Table 2. Atmospheric fundamental parameters obtained from Strömgren and Geneva photometry for the sample stars and their derived radial velocity.

\begin{tabular}{rrrrrr}
\hline \hline & \multicolumn{3}{c}{ Strömgren } & \multicolumn{3}{c}{ Geneva } \\
HD & $T_{\text {eff }}$ & $\log g$ & $T_{\text {eff }}$ & $\log g$ & $v_{\mathrm{r}}$ \\
& {$[\mathrm{K}]$} & {$[\mathrm{cgs}]$} & {$[\mathrm{K}]$} & {$[\mathrm{cgs}]$} & {$\left[\mathrm{km} \mathrm{s}^{-1}\right]$} \\
\hline 127929 & 7552 & 3.67 & 7361 & 3.94 & -18.9 \\
138918 & & & 7345 & 4.02 & -41.6 \\
143466 & 7380 & 3.96 & & & -13.4 \\
124675 & & & 7470 & 4.08 & -17.1 \\
124953 & & & 7388 & 4.38 & +5.3 \\
125161 & 7832 & 4.14 & 7755 & 4.46 & -16.9 \\
127762 & 7797 & 3.67 & 7581 & 3.97 & -31.6 \\
\hline
\end{tabular}

The $v_{\mathrm{r}}$, in $\mathrm{km} \mathrm{s}^{-1}$, has an error bar of about $4 \mathrm{~km} \mathrm{~s}^{-1}$.

The photometric and the adopted spectroscopic fundamental parameters are shown in Table 2 . We determined the abundance by fitting the core of the selected lines. The spectra were synthesised with Synth3 (Kochukhov 2007). The fitting procedure and the determination of the abundances was done in an iterative process in order to get better values for the abundances of the more blended lines. The error bar associated with each element is the standard deviation from the mean abundance of the selected lines of that element. For all the elements that were not analysed we adopted the solar abundance from Grevesse et al. (2007).

HD 138918, HD 124675, HD 127762 and possibly HD 124953 are binary stars (Hoffleit \& Jaschek 1991). No direct indication of the spectrum of the secondary star was found in our data, since its flux is probably below $5 \%$ of the primary's flux.

We measured the radial velocity $\left(v_{\mathrm{r}}\right)$ and $v \sin i$ from fitting the observed data with synthetic spectra. This analysis was based on about eighty lines and the typical uncertainty on $v_{\mathrm{r}}$ and $v \sin i$ is of the order of $4 \mathrm{~km} \mathrm{~s}^{-1}$ and $5 \%$, respectively.

\section{Results}

In this section we present the results of the abundance analysis obtained for each star in our sample. The pulsation characteristics of the individual targets also are briefly mentioned. Due to the brightness of most of the targets their photometric variation has not always been well studied, due to a lack of good comparison stars in the vicinity. Hence the information on the pulsation may not be fully reliable.

The derived abundances and the estimated uncertainties are given in Table 3 and shown in Fig. 11. The abundances of the whole sample of field $\delta$ Scuti stars appear to be quite dispersed, except for e.g. Si, Ti and Mn. The abundances of most of the elements (C, O, Mg, Si, $\mathrm{Ca}, \mathrm{Sc}, \mathrm{Cr}, \mathrm{Fe}$ and $\mathrm{Ni}$ ) appear to be close to solar, while $\mathrm{Na}, \mathrm{S}, \mathrm{Ti}, \mathrm{Mn}, \mathrm{Y}$ and $\mathrm{Ba}$ show a slight (but clear) overabundance.

\section{1. $H D 127929$}

The $\delta$ Scuti nature of HD 127929 (HR 5437, ER Dra) was discovered by Jiang \& Li (1988). The star has a pulsation amplitude of about 0.02 mag in $V$. Li \& Jiang (1992) found the main pulsational frequency to be $11.38993 \mathrm{c} / \mathrm{d}$. This frequency, 
Table 3. Abundances $\left(\log \left(N_{\mathrm{X}} / N_{\text {tot }}\right)\right)$ of the program stars.

\begin{tabular}{|c|c|c|c|c|c|c|c|c|c|}
\hline At.N. & Element & HD 127929 & HD 138918 & HD 143466 & $\begin{array}{c}\delta \text { Scuti stars } \\
\text { HD } 124675\end{array}$ & HD 124953 & HD 125161 & HD 127762 & $\begin{array}{r}\text { Solar } \\
\text { Abundances }\end{array}$ \\
\hline 6 & $\mathrm{C}$ & $-3.40(-; 1)$ & $-3.34(09 ; 2)$ & $-3.71(-; 1)$ & & $-3.78(06 ; 2)$ & $-3.62(09 ; 2)$ & $-3.44(-; 1)$ & -3.65 \\
\hline 8 & $\mathrm{O}$ & $-3.41(-; 1)$ & $-3.49(-; 1)$ & & & & & & -3.38 \\
\hline 11 & $\mathrm{Na}$ & $-5.76(06 ; 2)$ & $-5.57(10 ; 2)$ & & & $-5.65(01 ; 2)$ & & & -5.87 \\
\hline 12 & $\mathrm{Mg}$ & $-4.29(04 ; 4)$ & $-4.08(06 ; 4)$ & $-4.38(-; 1)$ & $-4.77(11 ; 3)$ & $-4.51(10 ; 5)$ & $-4.39(09 ; 2)$ & $-4.29(01 ; 2)$ & -4.51 \\
\hline 14 & $\mathrm{Si}$ & $-4.59(-; 1)$ & $-4.64(-; 1)$ & $-4.60(-; 1)$ & $-4.50(-; 1)$ & $-4.54(-; 1)$ & $-4.36(-; 1)$ & $-4.61(-; 1)$ & -4.53 \\
\hline 16 & $\mathrm{~S}$ & $-4.75(03 ; 2)$ & $-4.58(06 ; 2)$ & $-4.68(08 ; 2)$ & $-4.51(-; 1)$ & $-4.63(07 ; 5)$ & $-4.28(05 ; 2)$ & $-4.37(04 ; 2)$ & -4.90 \\
\hline 20 & $\mathrm{Ca}$ & $-5.54(11 ; 7)$ & $-5.35(14 ; 9)$ & $-5.52(01 ; 2)$ & $-5.72(-; 1)$ & $-5.85(09 ; 5)$ & $-5.73(07 ; 6)$ & $-5.66(10 ; 5)$ & -5.73 \\
\hline 21 & $\mathrm{Sc}$ & $-8.91(11 ; 3)$ & $-9.09(04 ; 2)$ & $-8.76(04 ; 2)$ & $-8.84(15 ; 3)$ & $-9.24(13 ; 4)$ & $-8.99(01 ; 2)$ & $-8.84(07 ; 3)$ & -8.99 \\
\hline 22 & $\mathrm{Ti}$ & $-6.98(11 ; 4)$ & $-6.93(13 ; 7)$ & $-6.69(13 ; 2)$ & $-6.89(01 ; 2)$ & $-6.86(13 ; 7)$ & $-6.77(11 ; 7)$ & $-7.04(09 ; 4)$ & -7.14 \\
\hline 24 & $\mathrm{Cr}$ & $-6.36(07 ; 3)$ & $-6.06(12 ; 3)$ & $-6.02(-; 1)$ & $-6.84(04 ; 3)$ & $-6.26(23 ; 4)$ & $-6.49(19 ; 5)$ & $-6.48(09 ; 3)$ & -6.40 \\
\hline 25 & $\mathrm{Mn}$ & $-6.51(-; 1)$ & $-6.20(09 ; 2)$ & & & $-6.51(05 ; 6)$ & & $-6.36(-; 1)$ & -6.65 \\
\hline 26 & $\mathrm{Fe} I$ & $-4.44(05 ; 26)$ & $-4.26(10 ; 40)$ & $-4.54(06 ; 16)$ & $-4.85(11 ; 27)$ & $-4.38(08 ; 65)$ & $-4.56(08 ; 31)$ & $-4.58(11 ; 29)$ & \\
\hline 26 & $\mathrm{Fe}$ II & $-4.39(05 ; 5)$ & $-4.24(14 ; 3)$ & $-4.56(-; 1)$ & $-4.71(-; 1)$ & $-4.35(15 ; 3)$ & $-4.55(07 ; 5)$ & $-4.55(12 ; 5)$ & \\
\hline 26 & $\mathrm{Fe}$ & $-4.43(06 ; 31)$ & $-4.26(10 ; 43)$ & $-4.54(06 ; 17)$ & $-4.85(12 ; 28)$ & $-4.37(08 ; 68)$ & $-4.55(08 ; 36)$ & $-4.58(11 ; 34)$ & -4.59 \\
\hline 28 & $\mathrm{Ni}$ & $-5.92(11 ; 2)$ & $-5.53(11 ; 4)$ & & $-6.24(-; 1)$ & $-5.73(09 ; 5)$ & $-5.92(07 ; 3)$ & $-6.04(08 ; 2)$ & -5.81 \\
\hline 39 & $\mathrm{Y}$ & $-9.57(-; 1)$ & $-9.49(11 ; 2)$ & & $-9.73(-; 1)$ & $-9.36(-; 1)$ & $-9.47(-; 1)$ & $-9.22(-; 1)$ & -9.83 \\
\hline 56 & $\mathrm{Ba}$ & $-9.49(-; 1)$ & $-9.37(-; 1)$ & $-9.19(-; 1)$ & $-9.66(-; 1)$ & $-8.91(-; 1)$ & $-9.27(-; 1)$ & $-9.35(-; 1)$ & -9.87 \\
\hline & $\overline{T_{\text {eff }}}$ & 7600 & 7800 & 7380 & 7500 & 7600 & 7700 & 7800 & \\
\hline & $\log g$ & 3.70 & 3.50 & 3.90 & 4.00 & 4.10 & 4.45 & 3.75 & \\
\hline & $v_{\text {mic }}$ & 2.7 & 2.6 & 2.7 & 2.7 & 2.6 & 2.6 & 2.6 & \\
\hline & $v \sin i$ & 73 & 88 & 136 & 121 & 82 & 131 & 121 & \\
\hline & $\sigma_{\mathrm{abn}}$ & 0.23 & 0.23 & 0.26 & 0.25 & 0.23 & 0.26 & 0.25 & \\
\hline
\end{tabular}

The estimated internal errors in units of $0.01 \mathrm{dex}$ and the number of selected lines are given in parentheses. Abundances obtained from one line have no error (-). The uncertainties on the fundamental parameters, $T_{\text {eff }}, \log g$, and $v_{\text {mic }}$ are $200 \mathrm{~K}, 0.22$ dex and $0.7 \mathrm{~km} \mathrm{~s}^{-1}$. The uncertainty on $v \sin i$ is 5\%. The estimated uncertainty on the abundances $\left(\sigma_{\mathrm{abn}}\right)$, including contributions from the high $v \sin i$ and uncertainties on $T_{\text {eff }}$ and $v_{\text {mic }}$, are given in the last row of the table.

combined with another frequency at $8.5367 \mathrm{c} / \mathrm{d}$ found by Paparo et al. (1990) suggests that HD 127929 is a $\delta$ Scuti star pulsating in the radial fundamental and radial first overtone modes.

The analysed spectrum of this star is the sum of two consecutive spectra with equal exposure time. This was done to increase the signal-to-noise ratio and to avoid saturating the CCD. The abundances determined for this star seem to be almost solar for all elements, except for a slight Ba overabundance.

\section{2. $H D 138918$}

HD 138918 (HR 5789, $\delta$ Ser) is part of a multiple system consisting of a close binary (Muller 1950) with a separation angle of $4.000 \pm 0.001$ arcsec (Alzner 1998; Prieur et al. 2002), and two other stars of magnitude 13 and 14 in $V$. The primary is an A9V star with 4.25 mag in $V(\delta$ Ser $)$ and its companion an A7V star with 5.2 mag in $V$ (Baize \& Petit 1989).

López de Coca \& Rolland (1987) found two main frequencies at $6.4227 \mathrm{c} / \mathrm{d}$ and $7.8869 \mathrm{c} / \mathrm{d}$, which were identified as the first and second overtone, with a ratio of 0.814 . The star is one of the brightest $\delta$ Scuti stars in the sky (Breger 2008), which will be suitable for photometric monitoring with the BRITE microsatellite (Kaiser et al. 2008). It has a pulsation amplitude of about 0.04 mag in $V$.

There is a large difference between the parameters obtained from photometry $\left(T_{\text {eff }}=7345 \mathrm{~K} ; \log g=4.02\right)$ and the adopted spectroscopic parameters $\left(T_{\text {eff }}=7800 \mathrm{~K} ; \log g=3.50\right)$. Figure 4 shows the correlation with excitation potencial of the abundances of Fe I (circles) and Fe II (stars) lines adopting the fundamental parameters obtained from photometry (upper panel) and from spectroscopy (lower panel). The positive trend in the upper panel of Fig. 4 is mainly due to the presence of two lines with

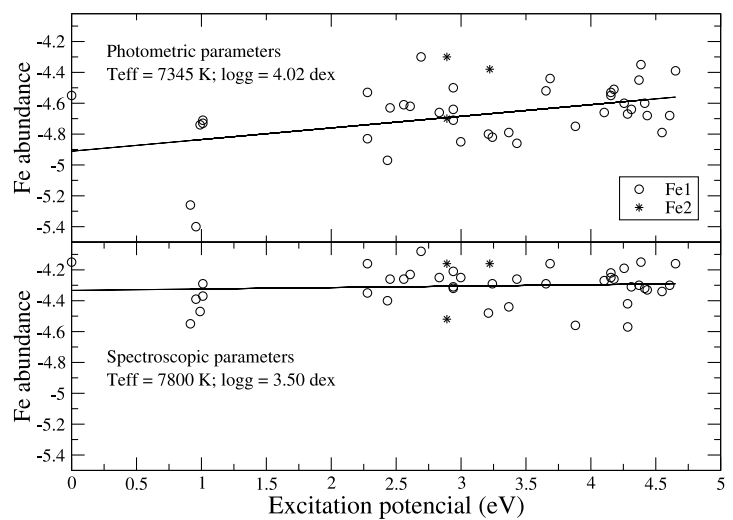

Fig. 4. Correlation between Fe I (circles) and Fe II (stars) abundances and excitation potencial adopting photometric (upper panel) and spectroscopic (lower panel) parameters for HD 138918.

low excitation potencial, which are very sensitive to changes in temperature $^{2}$.

We are not able to explain the reason for the discrepancy between the parameters derived photometrically and spectroscopically. It could be that this difference is due to the effects of the secondary, of which we do not see any lines, but this is just a speculation.

The analysis of this star yields a general overabundance of about 0.3 dex for all the elements, while $\mathrm{O}, \mathrm{Si}$ and $\mathrm{Sc}$ are consistent with the solar abundance.

\footnotetext{
${ }^{2}$ Note that the scatter of the points is significantly lower for the higher temperature as determined from spectroscopy.
} 


\section{3. $H D 143466$}

The variability of HD 143466 (HR 5960, CL Dra) was discovered by Breger (1969), who classified the star as a $\delta$ Scuti variable. DuPuy \& Burgoyne (1983) found a main frequency $f_{1}$ at $14.7929 \mathrm{c} / \mathrm{d}$ (which could also be $14.5985 \mathrm{c} / \mathrm{d}$ ) and a secondary frequency $f_{2}$ at $20.2840 \mathrm{c} / \mathrm{d}$. The star has a pulsation amplitude of about $0.01 \mathrm{mag}$ in $V$.

Due to the rather high rotational velocity of the $\operatorname{star}(v \sin i=$ $136 \mathrm{~km} \mathrm{~s}^{-1}$ ) it was only possible to derive abundances of the main elements. Except for an overabundance of $\mathrm{Ti}, \mathrm{Cr}$ and $\mathrm{Ba}$, the other elements show almost solar abundances.

\section{4. $H D 124675$}

HD 124675 (HR 5329, $\kappa^{2}$ Boo) is a binary star and one component is known to be a $\delta$ Scuti pulsator of spectral type A8IV. The other component $\left(\kappa^{1}\right.$ Boo) is an F1V star. Bakos (1986) determined the period of the system to be 1790 days, and the semiamplitude of the velocity curve is $7.2 \mathrm{~km} \mathrm{~s}^{-1}$. Frandsen et al. (1995) found that the star shows $\delta$ Scuti-type pulsation with a few closely spaced modes. They found frequencies at $f_{1}=15.43 \mathrm{c} / \mathrm{d}, f_{2}=15.58 \mathrm{c} / \mathrm{d}, f_{3}=14.52 \mathrm{c} / \mathrm{d}, f_{4}=15.81 \mathrm{c} / \mathrm{d}$. The star has a pulsation amplitude (sum of all modes) of about $0.05 \mathrm{mag}$ in $V$. Their preliminary mode identification points towards the second overtone for the $f_{1}$ mode $(Q \simeq 0.016)$. The other modes may perhaps have $\ell$ values of 2 and 1 , but more observations and detailed modelling including the effects of rotation are needed to obtain a robust mode identification. Walker et al. (1987) and Kennelly et al. (1991) showed from line profile analysis that the star has non-radial pulsation modes. Thanks to the fast rotation of the star it is possible to detect modes with even high degrees. Kennelly et al. (1991) found that a mode with high $\ell(\simeq 12)$ in combination with a low-degree non-radial mode can explain the observed line profile variations. From high-quality data more non-radial pulsation modes could likely be found.

The abundances determined for HD 124675 are almost solar for all the elements, except of S (overabundant) and $\mathrm{Cr}$ and $\mathrm{Ni}$ (underabundant). Among the stars of our sample, HD 124675 shows the lowest average abundance.

\section{5. $H D 124953$}

HD 124953 (HR 5343, CN Boo) was discovered to show $\delta$ Scuti pulsation by Costa et al. (1979). They found a frequency of about $25 \mathrm{c} / \mathrm{d}$ which they identified through the $Q$ value of 0.025 as the first overtone radial mode. Given the pulsation, they concluded that the classification of HD 124953 as an Am star (Hoffleit \& Jaschek 1991) must be wrong, since Am stars are not supposed to pulsate (see Sect. 4.3). The star has a pulsation amplitude of about 0.03 mag in $V$. In the Bright Star Catalogue (Hoffleit \& Jaschek 1991) the star is marked as a suspected spectroscopic binary. Our spectrum does not show any feature that indicate this.

The error bar associated with the $\mathrm{Cr}$ abundance is quite large relative to the other elements. This is due to the difference in abundance found for Cr I and Cr II (we used two spectral lines for each ionisation stage). In particular one Cr II line ( 5280 ̊) deviates from the mean abundance. We checked the log $g f$ value of this line by comparing with a spectrum of the Sun, and it seems to be correct. An improper continuum normalisation could explain the discrepancy for this line.

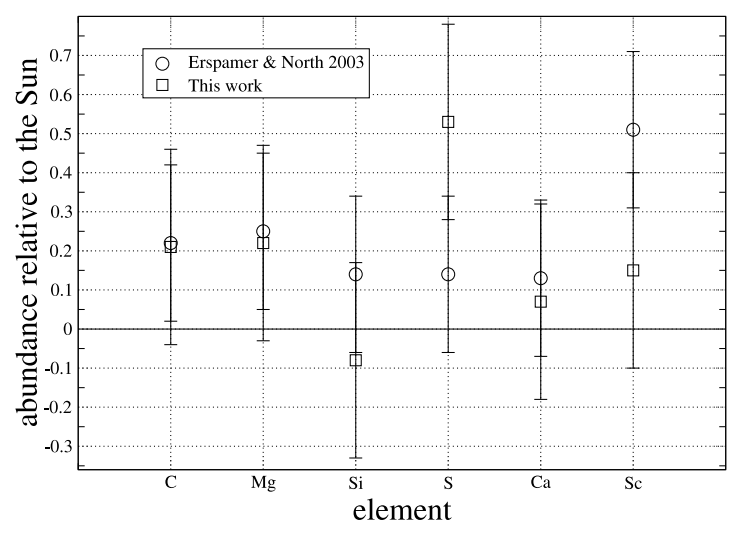

Fig. 5. Comparison between the abundances in HD 127762 derived by Erspamer \& North (2003) and this work.

HD 124953 shows a slight underabundance of $\mathrm{C}$ and $\mathrm{Ca}$, an underabundance of Sc, a slight overabundance of the Fe-peak elements and a clear overabundance of $\mathrm{Y}$ and $\mathrm{Ba}$. The other elements show almost solar abundances. This abundance pattern, typical of Am stars, will be discussed in detail in Sect. 4.3.

\section{6. $H D 125161$}

HD 125161 (HR 5350, ८ Boo) was discovered to be a $\delta$ Scuti star by Albert (1980). It was discussed by several authors (Kiss et al. 1999; Zhou 1999) showing pulsation in photometry, which can be fitted with one single period. Zhou (1999) found the main frequency to be $f_{1}=37.6804 \mathrm{c} / \mathrm{d}$ and also found a low-amplitude secondary peak at $f_{2}=36.4111 \mathrm{c} / \mathrm{d}$. The star has a pulsation amplitude of about $0.01 \mathrm{mag}$ in $V$.

Only S, Ti, Y and Ba appear to be overabundant, while all the other elements show solar abundances. As for HD 124953 (see Sect. 3.5), the error bar associated with the $\mathrm{Cr}$ abundance is large and for the same reason.

\section{7. $H D 127762$}

HD 127762 (HR 5435, $\gamma$ Boo) is one of "the magnificent seven", the seven brightest $\delta$ Scuti stars which are on the target list of the BRITE microsatellite (Breger 2008; Kaiser et al. 2008). The star is part of a multiple system of which the primary itself is found to be double by speckle interferometry with a separation of 0.069 arcsec (Hartkopf et al. 1997). The star was discovered as a short-period variable by Guthnick \& Prager (1914), with a pulsation amplitude of about $0.05 \mathrm{mag}$ in $V$. The star pulsates with a dominant mode at $f=21.28 \mathrm{c} / \mathrm{d}$.

Marilli et al. (1992) reported a possible detection of Ly- $\alpha$ emission in the star. From high-resolution spectra Kennelly et al. (1992) were the first to detect a high-degree, non-radial pulsation in HD 127762. Ventura et al. (2007) confirmed their result and found additional modes.

Most elements appear to have solar abundance. The elements S, Y and Ba appear to be overabundant, but the analysis is based on just one or two lines.

Erspamer \& North (2003) derived the fundamental parameters and abundances for HD 127762. They obtained $T_{\text {eff }}=7585$, $\log g=3.74, v_{\text {mic }}=3.1$ and $v \sin i=123$ and these parameters are in good agreement with our result. Erspamer \& North (2003) measured abundances relative to the Sun using the solar abundances by Grevesse \& Sauval (1998). Figure 5 shows the 


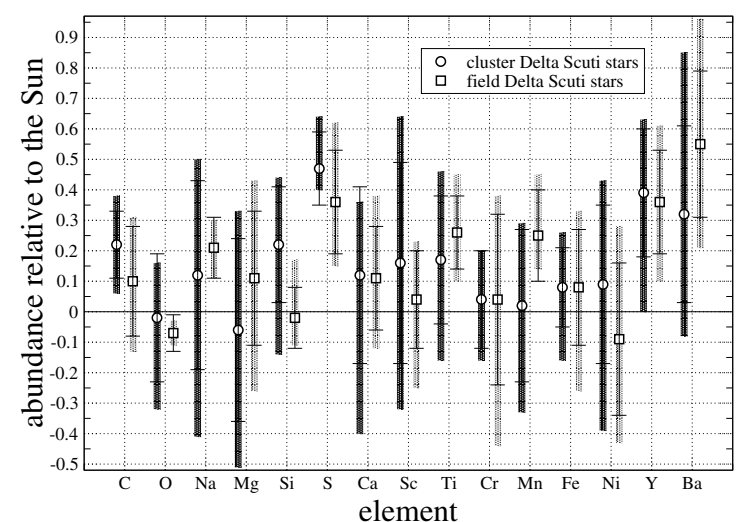

Fig. 6. Mean abundance, relative to the Sun, and abundance range (shaded areas) of the sample of field $\delta$ Scuti stars (squares) and cluster $\delta$ Scuti stars (circles). The error bars correspond to the standard deviation of the mean abundance.

comparison between the abundances derived by Erspamer \& North (2003), after the conversion to our adopted solar abundances, and this work. The abundances for all the plotted elements are in agreement within the errors.

\section{Discussion}

In the following section we will compare the results of the abundance analysis of seven field $\delta$ Scuti stars with recent results for stars belonging to the Praesepe cluster (Fossati et al. 2007, 2008). Furthermore, we will compare our results with results for four other field $\delta$ Scuti stars. Finally, we will discuss in detail the possible Am nature of the $\delta$ Scuti star HD 124953.

\subsection{Abundances in field and cluster $\delta$ Scuti stars}

We compared two samples of $\delta$ Scuti stars (i) belonging to the Praesepe cluster, all with a common and well defined age (708 \pm 300 Myr, González-Garcı et al. 2006); and (ii) field $\delta$ Scuti stars. In Fig. 6 we show the mean abundance for each analysed element of the two samples and their abundance range (indicated by the dashed area). Note that the two samples of stars are comparable since they are composed of almost the same number of stars (eight stars in the cluster sample and seven in the field sample) with similar spectral type.

The abundances of the two samples are close to solar with clear overabundances of S, Y and Ba. In HD 124953 we find evidence for the star being an Am star, and we will discuss it separately in Sect. 4.3. In the other stars we do not find abundance patterns typical for chemically peculiar stars.

The abundance patterns of the two samples appear to be quite similar. To have a statistically more solid sample of stars we decided to merge the two samples. To test the validity of merging them we carried out a paired $t$-test. The test measures the probability that the samples are the same, and it was done separately for the 15 elements. The $t$ values and corresponding probabilities that the two samples refer to the same statistical population are provided in Table 4 . The bottom row of the table refers to the overall $t$-test merging all elements. The result, $t=0.06$, corresponds to a probability of $95 \%$ that the two samples agree in their overall distribution of abundances. We consider this a sufficient argument for a common treatment of cluster and field $\delta$ Scuti stars.
Table 4. Statistical comparison of the abundances of 15 elements for cluster and field $\delta$ Scuti stars.

\begin{tabular}{|c|c|c|c|c|c|c|c|c|}
\hline \multirow[b]{2}{*}{ El. } & \multicolumn{3}{|c|}{$\overline{\text { Cluster }}$} & \multicolumn{3}{|c|}{$\overline{\text { Field }}$} & \multirow[t]{2}{*}{$t$} & \multirow[t]{2}{*}{$\overline{\mathrm{Pr}}$} \\
\hline & \# & mean & $\mathrm{rms}$ & \# & mean & $\mathrm{rms}$ & & \\
\hline $\mathrm{C}$ & 7 & +0.22 & 0.28 & 6 & +0.10 & 0.30 & 0.753 & 0.467 \\
\hline $\mathrm{O}$ & 4 & -0.02 & 0.33 & 2 & -0.07 & 0.23 & 0.196 & 0.854 \\
\hline $\mathrm{Na}$ & 7 & +0.12 & 0.41 & 3 & +0.21 & 0.25 & 0.346 & 0.738 \\
\hline $\mathrm{Mg}$ & 8 & -0.06 & 0.40 & 7 & +0.11 & 0.33 & 0.858 & 0.407 \\
\hline $\mathrm{Si}$ & 8 & +0.22 & 0.33 & 7 & -0.02 & 0.26 & 1.511 & 0.155 \\
\hline S & 5 & +0.47 & 0.29 & 7 & +0.36 & 0.30 & 0.672 & 0.517 \\
\hline $\mathrm{Ca}$ & 8 & +0.12 & 0.40 & 7 & +0.11 & 0.29 & 0.092 & 0.928 \\
\hline $\mathrm{Sc}$ & 8 & +0.16 & 0.43 & 7 & +0.04 & 0.29 & 0.605 & 0.556 \\
\hline $\mathrm{Ti}$ & 8 & +0.17 & 0.34 & 7 & +0.26 & 0.27 & 0.572 & 0.577 \\
\hline $\mathrm{Cr}$ & 7 & +0.04 & 0.31 & 7 & +0.04 & 0.37 & 0.016 & 0.988 \\
\hline $\mathrm{Mn}$ & 7 & +0.02 & 0.37 & 4 & +0.25 & 0.28 & 1.120 & 0.292 \\
\hline $\mathrm{Fe}$ & 8 & +0.08 & 0.30 & 7 & +0.08 & 0.31 & 0.001 & 0.999 \\
\hline $\mathrm{Ni}$ & 8 & +0.09 & 0.37 & 6 & -0.09 & 0.34 & 0.916 & 0.378 \\
\hline Y & 7 & +0.39 & 0.34 & 6 & +0.36 & 0.30 & 0.210 & 0.838 \\
\hline $\mathrm{Ba}$ & 8 & +0.32 & 0.39 & 7 & +0.55 & 0.34 & 1.179 & 0.260 \\
\hline & 108 & & & 90 & & & 0.060 & 0.952 \\
\hline
\end{tabular}

For each element, the number of stars, the mean abundance, and the standard error is given. The last two columns represent the $t$ values for a paired Student test and the resulting probabilities (Pr) that the two samples are taken from the same statistical population. The bottom row refers to the overall statistics for all elements.

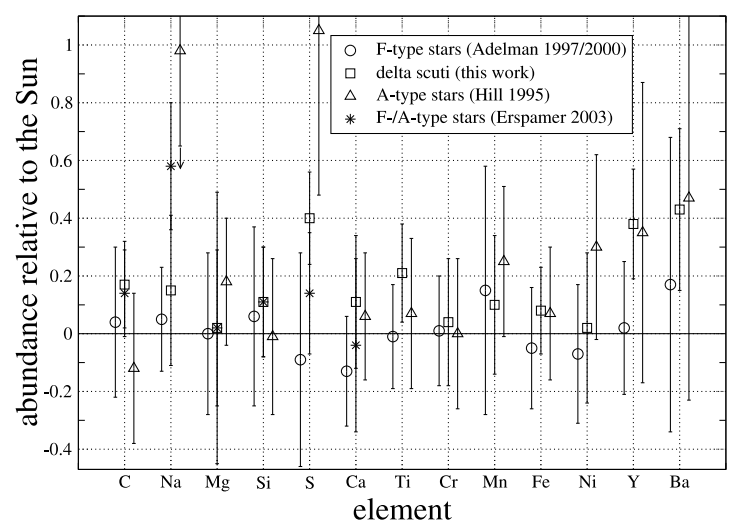

Fig. 7. Comparison of the mean abundances and standard deviations for four samples of stars. The Na abundance of the A-type stars (Hill 1995) is an upper limit.

In order to be able to confirm that $\delta$ Scuti stars in general have abundances that are typical of non-variable early F- and late A-type stars we have used three comparison samples. We took a sample of non-variable late F-type stars $\left(5\right.$ stars, $T_{\text {eff }} \sim$ $6800 \mathrm{~K})$, analysed by Adelman et al. (1997, 2000), non-variable early F- and late A-type stars (with a mean $T_{\text {eff }}$ corresponding to our sample; 11 stars, $T_{\text {eff }} \sim 7600 \mathrm{~K}$ ) analysed by Erspamer $\&$ North (2003) and of non-variable early A-type stars (15 stars, $\left.T_{\text {eff }} \sim 9300 \mathrm{~K}\right)$, analysed by Hill (1995). In Fig. 7 we compare the mean abundances and uncertainties for the four samples. For most elements the abundances are close to solar, although we find slightly higher abundances of $\mathrm{S}, \mathrm{Y}$ and $\mathrm{Ba}$. In our sample we find a much lower abundance of $\mathrm{Na}$ compared to the sample from Erspamer \& North (2003). This is probably due to the fact that the $\mathrm{Na}$ lines used by them ( $\mathrm{Na}$ I doublet at $~ 5889$ and $\sim 5895 \AA$ ) to derive the Na abundance are known to be affected by strong non-LTE effects that would lead to a higher abundance. We avoided the use of these two lines. 


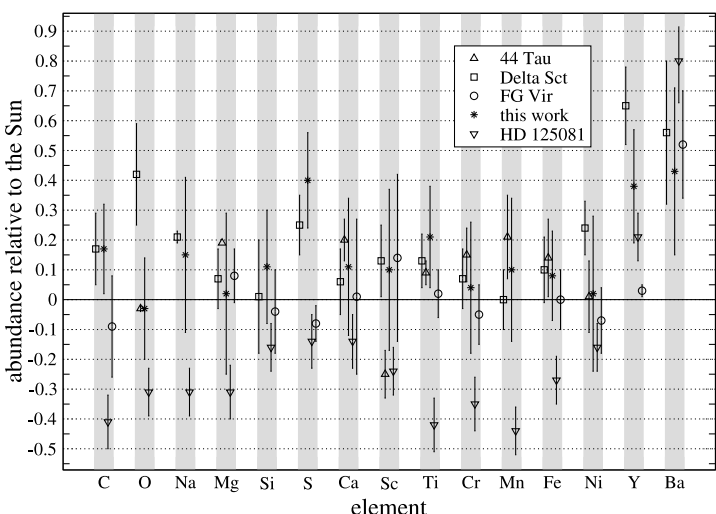

Fig. 8. Comparison of the mean abundance pattern of our sample with four other $\delta$ Scuti stars: FG Vir, $\delta$ Sct, 44 Tau and HD 125081. The adopted error bars for the single stars are taken from the original papers.

\subsection{Comparison with single $\delta$ Scuti stars}

We compared the abundance pattern in our sample with results for four single field $\delta$ Scuti stars: FG Vir (Mittermayer \& Weiss 2002), $\delta$ Sct (Yushchenko et al. 2005), 44 Tau (Zima et al. 2007) and HD 125081 (Bruntt et al. 2008). In Fig. 8 we compare the abundances of these four objects.

The mean effective temperature of our sample is $7600 \mathrm{~K}$. The comparison stars are slightly cooler: HD 125081 has $T_{\text {eff }}=$ $6400 \mathrm{~K}, \delta$ Sct and 44 Tau have $T_{\text {eff }}=7000 \mathrm{~K}$, and FG Vir has $T_{\text {eff }}=7425 \mathrm{~K}$. The results presented in Figs. 7 and 8 show that there is no obvious temperature effect, although the coolest star, HD 125081, has low abundance of both light and Fe-peak elements. To be able to confirm that this is a temperature effect, more comparison stars would be needed.

\subsection{HD 124953: an Am-type $\delta$ Scuti star?}

HD 124953 was first classified as a metallic-line star by Walker (1966), based on photometric indices. This has been confirmed by several other studies (e.g. Hauck 1973). An abundance analysis to give a definite classification as an Am star was never performed. Bertaud \& Floquet (1967), however, concluded it was a normal F0IV star in which the Ca I lines are weaker than usual.

It is well-established that Am stars show underabundances of $\mathrm{C}, \mathrm{N}, \mathrm{O}, \mathrm{Ca}$ and $\mathrm{Sc}$ and overabundances of the Fe-peak elements, Y, Ba and of the rare earths elements (Adelman et al. 1997; Fossati et al. 2007).

Charbonneau \& Michaud (1991) gave a rotational velocity limit of $90 \mathrm{~km} \mathrm{~s}^{-1}$ above which diffusion processes cannot cause Am peculiarities. Within an error of 5\% the $v \sin i$ of HD 124953 is close to this limit, but certainly below $90 \mathrm{~km} \mathrm{~s}^{-1}$. If the star is viewed equator on, the rotational velocity would be low enough to allow the star to be a mild Am. Fossati et al. (2008) have confirmed observationally that the abundances of the elements characteristic for Am stars are strongly related to the rotational velocity. In Fig. 9 we compare the abundances of HD 124953 with those obtained for the fastest rotator analysed by Fossati et al. (2007): HD 73818. This Am star has a $v \sin i$ of $66 \mathrm{~km} \mathrm{~s}^{-1}$ and an effective temperature ( $\left.T_{\text {eff }}=7230 \mathrm{~K}\right)$ comparable to HD 124953. The Am star HD 73818 is a member of the Praesepe cluster.

HD 124953 shows underabundances of C, Ca, Sc and overabundances of the Fe-peak elements, $\mathrm{Y}$ and $\mathrm{Ba}$, which is typical for Am-type stars. Note that the high abundance of $\mathrm{Si}$ in HD 73818 was not seen in the other Am stars in the Praesepe sample (see Fossati et al. 2007). The only relevant difference

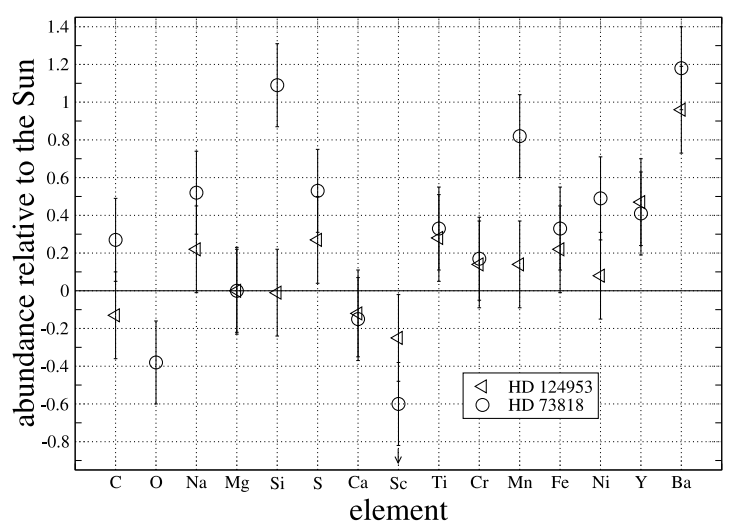

Fig. 9. Abundances of HD 124953 are compared with HD 73818. The latter is a fast-rotating Am star belonging to the Praesepe cluster.

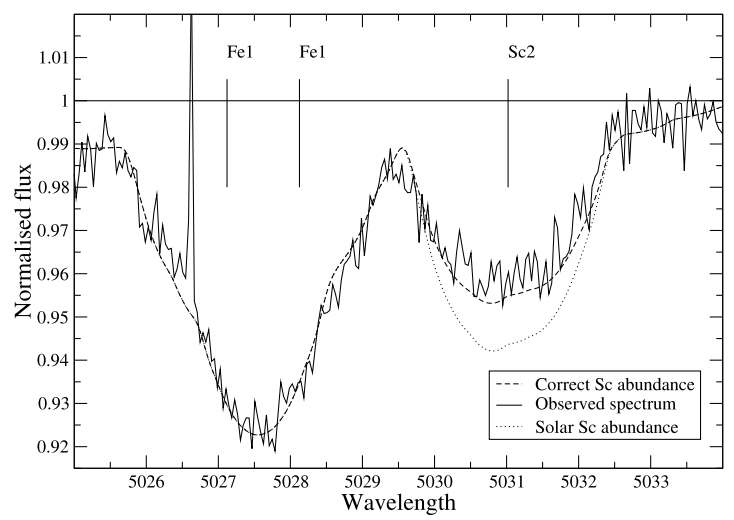

Fig. 10. Observed spectrum of HD 124953 and synthetic spectra around the Sc II line at 5031.2 $\AA$. The dotted line is for solar abundance of Sc and the dashed line is for the derived abundance.

between the two objects is the Sc abundance. According to Fossati et al. (2008) the Sc underabundance increases with the rotational velocity in contrast with predictions from diffusion models (see Talon et al. 2006; Leblanc \& Alecian 2008). If Sc behaved like the other underabundant elements, as predicted by diffusion models, its abundance should be close to the relative abundance of Ca. This is the case for HD 124953, but not for HD 73818. Figure 10 shows the observed and synthesised Sc II line at 5031.021 ^ with solar abundance (dotted line) and the derived Sc abundance (dashed line). It is clear that the solar Sc abundance is too high. The synthetic spectrum for the fitted abundance is shown in Fig. 10 and it is 0.43 dex less than the solar value.

The diffusion processes that lead to chemical peculiarities inhibit $\delta$ Scuti type pulsation (see Kurtz 2000, for Ap and Am stars). However, there are some important exceptions. Classical Am stars that have been proven to be low-amplitude $\delta$ Scuti stars do exist, such as HD 1097 (Kurtz 1989), HD 13038 (Martinez et al. 1999a), and HD 13079 (Martinez et al. 1999b). Recently, more Ap and Am stars showing pulsation were reported by Joshi et al. (2006). Henry \& Fekel (2005) and King et al. (2008) reported on the discoveries of Am stars showing hybrid pulsations, both in the $\gamma$ Doradus and the $\delta$ Scuti pulsation period ranges.

The Am character of a star can be derived from the metallicity index $\left(\delta m_{1}\right)$ and luminosity index $\left(\delta c_{1}\right)$, as was done for HD 124953. Both indices are sensitive to the strong lineblanketing found in the Am and Ap stars. The analysis here 


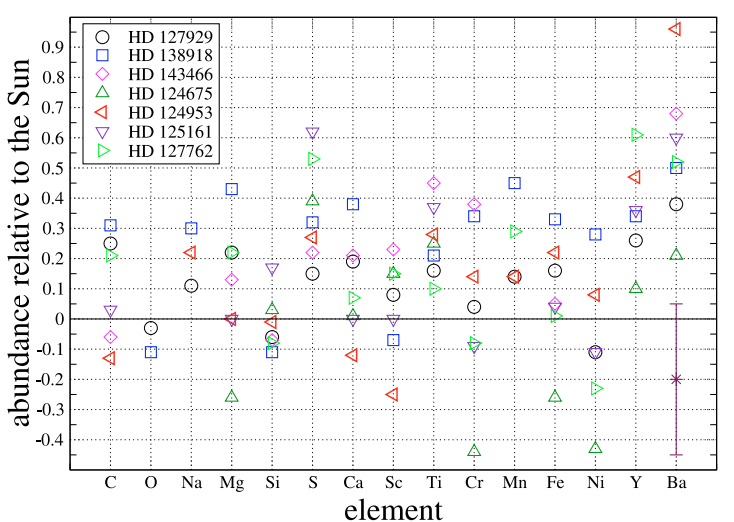

Fig. 11. Elemental abundances relative to the Sun for the program stars. The solar abundances are taken from Grevesse et al. (2007). In order to show a more readable figure, the error bars (Table 3) are omitted. The abundances of the Am- $\delta$ Scuti star are highlighted in red. The error bar associated to the star symbol (bottom right of the figure) shows the typical uncertainty associated with the plotted abundances.

presented for HD 124953 has confirmed its photometric classification as Am star. This is probably the first abundance analysis of a pulsating Am star.

Nearly all Am stars are found to occur in binary systems with orbital periods between 1 and 10 days in which the rotation and orbital periods are locked. As a result of the relatively slow rotation, chemical peculiarities can be found in these stars (Kurtz 2000). For a supposedly pulsating Am star in a binary, it is therefore important to prove that the pulsating star is really the Am star and not the other component in the system. A historic example is $32 \mathrm{Vir}$, for which claims of $\delta$ Scuti pulsation in Am stars were dismissed by Kurtz et al. (1976). Given the binary nature of the star, they found no convincing evidence for the pulsation of a single classical Am star. Later it was found that the primary is in fact a pulsating $\rho$ Puppis star (Mitton \& Stickland 1997).

HD 124953 is marked as a suspected spectroscopic binary ("SB?") in the Bright Star Catalogue (Hoffleit \& Jaschek 1991). In our spectrum of the star, however, we see no evidence of a possible companion star, as already mentioned in Sect. 2. The radial velocity we find for the star $\left(5.3 \pm 4.0 \mathrm{~km} \mathrm{~s}^{-1}\right)$ is in agreement with those found in the literature ( 3 references in SIMBAD), and shows no evidence of any significant radial velocity variation. Hence, at this point we have no evidence for the binary nature of the star, and it remains a candidate as an Am star showing $\delta$ Scuti pulsations.

\section{Conclusions}

We obtained high-resolution, high signal-to-noise ratio spectra of seven bright field $\delta$ Scuti stars. Due to their brightness, these stars have in general not been studied in great detail photometrically. Hence, time-series photometry to study the pulsation frequencies and/or measure the phase lag in different colours for mode identification has not been carried out. The analysed stars are potential future targets for microsatellite projects such as BRITE (Kaiser et al. 2008), and we discussed briefly their pulsation properties.

We compared the abundance pattern of the seven field $\delta$ Scuti stars with a sample of $\delta$ Scuti stars belonging to the Praesepe cluster (Fossati et al. 2007, 2008). A $t$-test confirms that the abundance patterns of the two samples (field and cluster $\delta$ Scuti stars) are comparable, allowing us to build one homogeneous sample of fifteen early F- and late A-type $\delta$ Scuti stars.

We used the abundances of non-variable F- and A-type stars published by Adelman et al. (1997, 2000), Erspamer \& North (2003) and Hill (1995), to generate a typical abundance pattern for stars of similar spectral types. From a direct comparison, we showed that the mean abundance pattern of our sample of $\delta$ Scuti stars and of the non-variable F- and A-type stars are comparable. The sample that we built allows us to conclude that generally the abundances of $\delta$ Scuti stars are comparable to those of normal stars of similar spectral types. We also compared the abundance pattern in our sample with results for four single field $\delta$ Scuti stars from the literature, and found good general agreement.

HD 124953 has previously been classified as a metallic-line star based on photometric indices. Our abundance analysis confirms this classification. It would be worthwhile to follow up on this star with photometric and spectroscopic data to better constrain the pulsation characteristics and the possible binarity of which there is no evidence from our spectrum. In case of confirmed binarity it would be necessary to prove that the Am star pulsates and not the secondary component.

Acknowledgements. We thank Michel Breger for pleasant and fruitful discussions and James Silvester for the english revision of the manuscript. We gratefully acknowledge the referee Hans Bruntt for useful suggestions. L.F. has received support from the Austrian Science Foundation (FWF project P17890-N2). K.K. acknowledges funding through the Austrian Science Foundation (FWF project T395). This paper is based on observations obtained using the SOPHIE spectrograph at the Observatoire de Haute-Provence (France). We acknowledge also the OPTICON program (Ref number: 2007/011) for financial support given to the observing run.

\section{References}

Adelman, S. J., Caliskan, H., Kocer, D., \& Bolcal, C. 1997, MNRAS, 288, 470 Adelman, S. J., Caliskan, H., Kocer, D., Cay, I. H., \& Gokmen Tektunali, H. 2000, MNRAS, 316, 514

Albert, J. 1980, Journal of the American Association of Variable Star Observers (JAAVSO), 9, 20

Alzner, A. 1998, A\&AS, 132, 237

Bagnulo, S., Landstreet, J. D., Mason, E., et al. 2006, A\&A, 450, 777

Baize, P., \& Petit, M. 1989, A\&AS, 77, 497

Bakos, G. A. 1986, AJ, 91, 1416

Bertaud, C., \& Floquet, M. 1967, J. Obs., 50, 425

Breger, M. 1969, ApJS, 19, 99

Breger, M. 2000, in $\delta$ Scuti stars (Review), ed. M. Breger, \& M. Montgomery, ASP Conf. Ser., 220, 3

Breger, M., Beck, P., Lenz, P., et al. 2006, A\&A, 455, 673

Breger, M. 2008, Commun. Asteroseis., 152, 97

Bruntt, H., De Cat, P., \& Aerts, C. 2008, A\&A, 478, 487

Charbonneau, P., \& Michaud, G. 1991, ApJ, 370, 693

Costa, V., Garrido, R., \& Saez, M. 1979, Inf. Bull. Variable Stars, 1584, 1

DuPuy, D. L., \& Burgoyne, L. G. 1983, PASP, 95, 71

Erspamer, D., \& North, P. 2003, A\&A, 398, 1121

Fossati, L., Bagnulo, S., Monier, R., et al. 2007, A\&A, 476, 911

Fossati, L., Bagnulo, S., Landstreet, J., et al. 2008, A\&A, 483, 891

Frandsen, S., Jones, A., Kjeldsen, H., et al. 1995, A\&A, 301, 123

González-Garcá, B. M., Zapatero Osorio, M. R., Béjar, V. J. S., et al. 2006, A\&A, 460, 799

Grevesse, N., \& Sauval, A. J. 1998, Space Sci. Rev., 85, 161

Grevesse, N., Asplund, M., \& Sauval, A. J. 2007, Space Sci. Rev., 130, 105

Guthnick, P., \& Prager, R. 1914, Photoelektrische untersuchungen an spektroskopischen Doppelsternen und an Planeten (Berlin: Ferd. Dummlers Verlagsbuchhandlung)

Hartkopf, W. I., McAlister, H. A., Mason, B. D., et al. 1997, AJ, 114, 1639

Hauck, B. 1973, A\&AS, 10, 385

Henry, G. W., \& Fekel, F. C. 2005, AJ, 129, 2026

Hill, G. M. 1995, A\&A, 294, 536

Hoffleit, D., \& Jaschek, C. 1991, The bright star catalogue, 5th revised edition, Yale University Observatory 
Jiang, S.-Y., \& Li, Z.-P. 1988, Astron. Circ., 22, 1

Joshi, S., Mary, D. L., Martinez, P., et al. 2006, A\&A, 455, 303

Kaiser, A., Zwintz, K., \& Weiss, W. W. 2008, Comm. Asteroseis., 152, 89

Kennelly, E. J., Walker, G. A. H., \& Hubeny, I. 1991, PASP, 103, 1250

Kennelly, E. J., Yang, S., Walker, G. A. H., \& Hubeny, I. 1992, PASP, 104, 15

King, H., Matthews, J. M., Rowe, J. F., et al. 2008, Comm. Asterosis., submitted [arXiv:0706.1804]

Kiss, L. L., Alfaro, E. J., Bakos, G., Csak, B., \& Szatmary, K. 1999, Informational Bulletin on Variable Stars, 4698, 1

Kochukhov, O. 2007, Spectrum synthesis for magnetic, chemically stratified stellar atmospheres, Physics of Magnetic Stars, 109, 118

Kunzli, M., North, P., Kurucz, R. L., \& Nicolet, B. 1997, A\&AS, 122, 51

Kupka, F., Piskunov, N. E., Ryabchikova, T. A., Stempels, H. C., \& Weiss, W. W. 1999, A\&AS, 138, 119

Kurtz, D. W. 1989, MNRAS, 238, 1077

Kurtz, D. W. 2000, Pulsation of Chemically Peculiar and Pre-Main Sequence Stars in the $\delta$ Scuti Instability Strip, Delta Scuti and Related Stars, ed. M. Breger, \& M. Montgomery, ASP Conf. Ser., 210, 287

Kurtz, D. W., Breger, M., Evans, S. W., \& Sandmann, W. H. 1976, ApJ, 207, 181

Landstreet, J. D., Bagnulo, S., Andretta, V., et al. 2007, A\&A, 470, 685

Landstreet, J. D., Silaj, J., Andretta, V., et al. 2008, A\&A, 481, 465

Leblanc, F., \& Alecian, G. 2008, A\&A, 477, 243

Li, Z., \& Jiang, S. 1992, A\&AS, 93, 87

López de Coca, P., \& Rolland, A. 1987, Inf. Bull. Variable Stars, 3108, 1

López de Coca, P., Rolland, A., Garrido, R., \& Rodriguez, E. 1990, A\&AS, 169, 211

Marilli, E., Catalano, S., Freire Ferrero, R., \& Gouttebroze, P. 1992, A\&A, 265, 643
Martinez, P., Ashoka, B. N., Kurtz, D. W., Gupta, S. K., \& Chaubey, U. S. 1999a, Inf. Bull. Variable Stars, 4677, 1

Martinez, P., Kurtz, D. W., Ashoka, B. N., et al. 1999b, MNRAS, 309, 871

Mittermayer, P., \& Weiss, W. W. 2003, A\&A, 407, 1097

Mitton, J., \& Stickland, D. J. 1997, MNRAS, 186, 189

Muller, P. 1950, Journal des Observateurs, 33, 105

Napiwotzki, R., Schoenberner, D., \& Wenske, V. 1993, A\&A, 268, 653

Paparo, M., Jiang, S.-Y., Li, Z.-P., \& Kollath, Z. 1990, Inf. Bull. on Variable Stars, 3451, 1

Piskunov, N. E., Kupka, F., Ryabchikova, T. A., Weiss, W. W., \& Jeffery, C. S. 1995, A\&AS, 112, 525

Prieur, J.-L., Koechlin, L., Ginestet, N., et al. 2002, ApJS, 142, 95

Ryabchikova, T. A., Piskunov, N. E., Stempels, H. C., Kupka, F., \& Weiss, W. W. 1999, Phis. Scr., T83, 162

Rodríguez, E., López-González, M. J., \& López de Coca, P. 2000, A\&AS, 144, 469

Shulyak, D., Tsymbal, V., Ryabchikova, T., Stütz, Ch., \& Weiss, W. W. 2004, A\&A, 428, 993

Talon, S., Richard, O., \& Michaud, G. 2006, ApJ, 645, 634

Vauclair, G., Vauclair, S. \& Michaud, G. 1978, ApJ, 223, 920

Ventura, R., Catanzaro, G., Christensen-Dalsgaard, J., di Mauro, M. P., \& Paternò, L. 2007, MNRAS, 381, 1647

Walker, E. N. 1966, The Observatory, 86, 154

Walker, G. A. H., Yang, S., \& Fahlman, G. G. 1987, ApJ, 320, L139

Yushchenko, A., Gopka, V., Kim, C., et al. 2005, MNRAS, 359, 865

Zhou, A.-Y. 1999, Delta Scuti Star Newsletter, 13, 7

Zima, W., Lehmann, H., Stütz, C., Ilyin, I. V., \& Breger, M. 2007, A\&A, 471, 237 